\title{
Estimativas de parâmetros genéticos para altura do posterior, peso e circunferência escrotal em bovinos da raça Nelore ${ }^{1}$
}

\author{
Marcos Jun Iti Yokoo ${ }^{2}$, Lucia Galvão de Albuquerque ${ }^{3}$, Raysildo Barbosa Lôbo ${ }^{4}$, Roberto \\ Daniel Sainz ${ }^{5}$, José Marques Carneiro Júnior ${ }^{2}$, Luiz Antônio Framartino Bezerra ${ }^{6}$, Fabiano \\ Rodrigues da Cunha Araujo ${ }^{7}$
}

\footnotetext{
1 Trabalho realizado com apoio do CNPq-Pronex e ANCP de Ribeirão-Preto-SP.

2 Pós-graduando do Programa de Genética e Melhoramento Animal da FCAV - Unesp/Jaboticabal, SP. Bolsista da FAPESP

${ }^{3}$ Departamento de Zootecnia - FCAV - Unesp/Jaboticabal, SP. Pesquisadora do CNPq.

4 Universidade para Desenvolvimento do Estado e da Região do Pantanal e Pesquisador Associado da ANCP de Ribeirão Preto-SP

${ }^{5}$ Departamento de Zootecnia da Universidade da Califórnia, Davis - CA 95616, USA.

${ }^{6}$ Analista de Sistemas do Departamento de Genética da USP de Ribeirão-Preto-SP.

${ }^{7}$ Aval Serviços Tecnológicos S/C, Uberaba-MG, Brasil.
}

RESUMO - Visando estimar parâmetros genéticos em bovinos, foram utilizados registros de pesos padronizados aos 120 , 210, 365, 450 e 550 dias de idade (P120, P210, P365, P450 e P550), altura do posterior mensurada próxima ao sobreano (ALT) e circunferências escrotais (CE) padronizadas aos 365, 450 e 550 dias de idade (CE365, CE450 e CE550). Os dados foram provenientes de animais machos e fêmeas, nascidos entre 1998 e 2003 em dez fazendas de seis estados brasileiros. Os componentes de (co)variância foram estimados pela metodologia REML em análises uni, bi e trivariadas, utilizando-se modelos animal. As estimativas de herdabilidade do efeito direto com os respectivos erros-padrão foram: ALT 0,63 (0,09), P120 0,25 (0,03), P210 0,34 (0,03), P365 0,45 (0,04), P450 0,48 (0,04), P550 0,49 (0,04), CE365 0,48 (0,04), CE450 $0,53(0,04)$ e CE550 0,42 (0,09). As correlações genéticas entre a ALT e as variáveis P120, P210, P365, P450 e P550 foram de 0,$68 ; 0,64 ; 0,53 ; 0,58$ e 0,59 , respectivamente. As associações genéticas do P120 com as CE ajustadas para peso e idade foram próximas de zero, entretanto, essas correlações foram positivas e moderadas, quando as CE foram ajustadas somente pela idade. As correlações genéticas da ALT com as CE, quando ajustadas para peso e idade, foram: -0,19 (CE365), -0,24 (CE450) e 0,00 (CE550). Utilizando um modelo que não incluiu o peso do animal como covariável, as correlações genéticas das CE com a ALT foram: 0,21 (CE365), 0,12 (CE450) e 0,39 (CE550). Essas estimativas indicam que as características de crescimento e CE apresentam variabilidade genética na raça Nelore, podendo ser incluídas em programas de melhoramento genético, e a seleção para peso em qualquer idade deve acarretar aumento na estatura dos animais. Desta forma, para obtenção de animais com tamanho e peso adequados ao sistema de produção, faz-se necessária a utilização de um índice de seleção aliando estas características.

Palavras-chave: Bos indicus, correlações genéticas, gado de corte, herdabilidade, peso padronizado

\section{Estimation of genetic parameters for hip height, weight and scrotal circumference in Nelore cattle}

ABSTRACT - Data consisting of standardized weights at 120, 210, 365, 450 and 550 days of age (W120, W210, W365, W450 and W550), hip height at 18 months of age (HH) and standardized scrotal circumferences (SC) at 365,450 and 550 days of age (SC365, SC450 and SC550) of Nelore animals born between 1998 and 2003, from ten ranches in six Brazilian States were used to estimate (co)variance components. Analyses were done by Restricted Maximum Likelihood methodology using single trait, two and three traits animal models. Heritability estimates of the direct effect for each trait and respective standard errors were: HH 0.63 (0.09), W120 0.25 (0.03), W210 0.34 (0.03), W365 0.45 (0.04), W450 0.48 (0.04), W550 0.49 (0.04), SC365 0.48 (0.04), SC450 0.53 (0.04) and SC550 0.42 (0.09). Genetic correlation estimates between HH and W120, W210, W365, W450, W550 were respectively 0.68, 0.64, 0.53, 0.58 and 0.59. Genetic correlations between W120 and SC adjusted for weight and age were close to zero, but positive and moderate when SC was adjusted only for age. Estimates of genetic correlation between HH and SC adjusted for weight and age were -0.19 (SC365), -0.24 (SC450) and 0.00 (SC550). The same parameter estimates when SC was adjusted only for age were 0.21 (SC365), 0.12 (SC450) and 0.39 (SC550). These results indicate genetic variability for growth traits and SC. Selection for weight at any age will increase stature. In order to obtain Nelore cattle with stature and weight suitable to the production system, both traits should be included in a selection index.

Key Words: beef cattle, Bos indicus, genetic correlations, heritability, standardized weight 


\section{Introdução}

A disponibilidade de estimativas de componentes de (co)variâncias e herdabilidade acuradas é essencial para o desenvolvimento de programas de melhoramento genético animal. Esses parâmetros genéticos são característicos de cada população e podem sofrer alterações, em conseqüência de seleção, mudanças no manejo, métodos e modelos de estimação, entre outras causas.

Os problemas reprodutivos são os principais limitantes da eficiência produtiva em bovinos de corte (Mattos \& Rosa, 1984). Entretanto, no Brasil, a maioria dos programas de melhoramento genético tem enfatizado as características de desempenho ponderal, sendo a circunferência escrotal (CE) uma das poucas características reprodutivas que compõem os índices de seleção adotados. A CE apresenta estimativas de correlações genéticas positivas com características de sêmen e crescimento (Knights et al., 1984) e com ocorrência de prenhez em fêmeas jovens (Silva et al., 2005) e negativas com idade à puberdade em machos e fêmeas e com idade ao primeiro parto em fêmeas (Bergmann et al., 1996; Dias et al., 2000; Pereira et al., 2000, 2002; Silva et al., 2000). Além disso, a CE tem estimativa de herdabilidade mais alta que as características reprodutivas de fêmeas, podendo ser utilizada como critério de seleção para as mesmas. As estimativas de herdabilidade da CE medidas ao ano e ao sobreano, em animais da raça Nelore, variam de 0,30 a 0,74 (Bergmann et al., 1996; Dias et al., 2003; Albuquerque et al., 2005; Sesana, 2005).

Vários criadores consideram apenas o peso e a CE do animal para seleção, o que pode conduzir a animais cada vez maiores. Essa prática pode não ser a mais apropriada em um sistema de criação extensivo, haja vista a inclusão da altura do posterior (ALT) nos critérios de seleção, como boa alternativa para controlar o tamanho dos animais. A característica ALT é de fácil mensuração e menos susceptível às variações de meio ambiente e reflete melhor o tamanho corporal quando comparada à medida de peso vivo do animal (Baker et al. 1998). Alguns autores (Vargas et al., 1998, 2000; Riley et al., 2002; Silva et al., 2003) estimaram herdabilidades de magnitude moderada à alta para a ALT, em animais zebuínos, variando de 0,30 a 0,87 .

As estimativas de correlações genéticas entre os pesos em diferentes idades e destes com a CE são freqüentes e, em geral, positivas e de magnitude média à alta (Knights et al., 1984; Alencar et al., 1993; Bergmann et al., 1996; Reyes et al., 1997; Siqueira et al., 2003). Na literatura, relata-se que a correlação genética entre ALT e peso é positiva e moderada (Silva et al., 2003).

No Brasil, trabalhos abordando associação genética entre a ALT e a CE e correlações entre CE, em diferentes idades nos animais zebuínos, são escassos. Entretanto, Bergmann et al. (1996), trabalhando com as CE nas idades de 365 e 450 dias, e Sesana (2005), estudando as CE medidas aos 9, 12, 15, 18 e 21 meses de idade, estimaram valores acima de 0,76 para as correlações genéticas entre as CE nas diferentes idades, em animais da raça Nelore.

Neste sentido, há necessidade de verificar não somente como essas características respondem à seleção em diferentes idades, mas também como a altura do posterior se associa às demais características que atualmente são consideradas nos programas de seleção. Desta forma, este trabalho foi conduzido com o objetivo de estimar herdabilidades para as características altura do posterior ao sobreano e circunferências escrotais ajustadas em diferentes idades, além de verificar as correlações genéticas entre elas e destas com pesos padronizados em diferentes idades.

\section{Material e Métodos}

Este trabalho foi desenvolvido com criadores da raça Nelore, associados ao Programa de Melhoramento Genético da Raça Nelore - Nelore Brasil (PMGRN) da Associação Nacional de Criadores e Pesquisadores de Ribeirão Preto SP (ANCP), conjuntamente com a Universidade Estadual Paulista (UNESP), Campus de Jaboticabal-SP.

Foram coletados dados da característica altura do posterior no sobreano (ALT), no período de 2002 a 2004, em 2.356 animais com idades entre 450 e 599 dias, machos e fêmeas, distribuídos em dez fazendas, provenientes dos estados de São Paulo, Mato Grosso do Sul, Mato Grosso, Minas Gerais, Paraná e Goiás. A ALT foi mensurada com o auxílio de um hipômetro, com o animal contido, tomando a medida da ponta do íleo até o solo, de acordo com o Beef Improvement Federation Guidelines (BIF, 1996).

Adicionalmente, foram avaliadas as medidas de peso padronizado aos 120, 210, 365, 450 e 550 dias de idade (P120, P210, P365, P450 e P550) e circunferências escrotais (CE) padronizadas aos 365, 450 e 550 dias de idade (CE365, CE450 e CE550) de animais nascidos entre os anos 1998 e 2003, em todos os rebanhos avaliados e constantes do arquivo de dados do PMGRN. Foram feitas mensurações trimestrais nesses rebanhos para as características de peso e circunferência escrotal. Os dados de pesos e das circunferências escrotais foram padronizados por:

Medida Padrão $=M_{a}+D \times d_{a}$, em que $M_{a}$ é a medida anterior à idade-padrão (peso ou circunferência escrotal); $d_{a}$, o número de dias compreendidos entre a mensuração anterior e a idade-padrão; e $D$ é dado por $D=\frac{M_{p}-M_{a}}{I_{p}-I_{a}}$, em 
que $D$ é a razão da diferença entre as medidas posterior e anterior pelo número de dias entre as duas medidas; $M_{p}$, a medida posterior à idade-padrão (peso ou circunferência escrotal); $I_{p}$, a idade na medida posterior à idade-padrão; e $I_{a}$, a idade na medida anterior à idade-padrão.

Para verificar como os efeitos de meio influenciam as características, inicialmente foram feitas as análises de variância pelo método dos quadrados mínimos, utilizando-se o procedimento GLM (SAS, 2000) e, com base nesta análise, definiram-se os grupos de contemporâneos (GC) e as covariáveis a serem incluídas no modelo. Na edição dos dados, eliminaram-se os animais cujas medidas estavam a três desvios-padrão acima ou abaixo da média de seu GC para cada característica. Foram excluídos GC que continham apenas animais filhos de um mesmo touro, além de GC com menos de três animais para as características ALT e CE550; para as características P365, P450 e P550, CE365 e CE450, os GC com menos de cinco animais; e para P120 e P210, os GC com menos de oito animais (Tabela 1).

Para formação dos GC, utilizaram-se as seguintes variáveis: fazenda, ano e bimestre de nascimento, sexo e lotes de manejos aos 120, 210, 365, 450 dias e na data de coleta dos dados. Os lotes de manejo eram formados por animais que permaneceram sob o mesmo manejo desde o nascimento até o respectivo momento em que foram tomadas as medidas.

Os componentes de variância foram estimados em análises uni, bi e trivariadas, usando modelos animal, por meio da metodologia REML (Máxima Verossimilhança Restrita), descrita por Patterson \& Thompson (1971), utilizando o programa computacional MTDFREML (Multiple Trait Derivative-Free Restricted Maximum Likelihood), desenvolvido por Boldman et al. (1995), que emprega o método da máxima verossimilhança restrita livre de derivadas. As estimativas de herdabilidades foram obtidas a partir das médias das variâncias genéticas aditivas e fenotípicas de todas as análises uni, bi e trivariadas, em que cada característica foi analisada. Para evitar a influência da seleção, utilizaram-se modelos bi e trivariados com o P120, para estimação dos parâmetros genéticos. Foi criada uma matriz de parentesco considerando até três gerações, incluindo todos os animais disponíveis, perfazendo um arquivo de pedigree contendo 36.133 animais. O modelo completo pode ser representado em notação matricial como:

$y=X \beta+Z_{1} a+Z_{2} m+W c+e$

em que y é o vetor das variáveis dependentes (pesos, ALT e circunferências escrotais); $X$, a matriz de incidência associando os efeitos fixos às variáveis dependentes; $\beta$, o vetor dos efeitos fixos; $Z_{1}$, a matriz de incidência de efeitos genéticos aditivos diretos para variáveis dependentes; $a$, o vetor de valores genéticos aditivos diretos dos animais; $\mathrm{Z}_{2}$, a matriz de incidência dos efeitos genéticos aditivos maternos (para P120, P210, P365 e P450); $m$, o vetor de valores genéticos aditivos maternos (para P120, P210, P365 e P450); W, a matriz do efeito de ambiente permanente da vaca (para P120, P210 e P365); c, o vetor de efeito do ambiente permanente da vaca (para P120, P210 e P365); e $e$, o vetor de efeitos residuais aleatórios.

Tabela 1 - Estrutura do arquivo dos dados, em bovinos da raça Nelore

Table 1 - Structure of the database of Nelore cattle used for the analyses

\begin{tabular}{|c|c|c|c|c|c|}
\hline $\begin{array}{l}\text { Característica } \\
\text { Trait }\end{array}$ & $\begin{array}{c}\text { № de animais } \\
N \text {. of animals }\end{array}$ & $\begin{array}{l}\text { Média (Desvio-padrão) } \\
\text { Mean (Standard deviation) }\end{array}$ & $\begin{array}{l}\text { № touros } \\
N \text {. of sires }\end{array}$ & $\begin{array}{l}\text { № vacas } \\
N \text {. of dams }\end{array}$ & $\begin{array}{l}\text { № } \mathrm{GC}^{10} \\
\text { N. of } C G^{10}\end{array}$ \\
\hline $\mathrm{CE} 65^{1}(\mathrm{~mm})$ & 5.377 & $209,68 \quad(22,60)$ & 372 & 3.917 & 169 \\
\hline $\mathrm{CE} 450^{2}(\mathrm{~mm})$ & 5.695 & $245,87 \quad(30,22)$ & 385 & 4.109 & 188 \\
\hline $\mathrm{CE} 550^{3}(\mathrm{~mm})$ & 1.771 & $276,78 \quad(34,44)$ & 204 & 1.543 & 129 \\
\hline $\mathrm{ALT}^{4}(\mathrm{~cm})$ & 2.356 & $136,06 \quad(5,04)$ & 226 & 2.308 & 250 \\
\hline $\mathrm{P} 120^{5}$ (kg) & 22.778 & $128,78 \quad(19,23)$ & 540 & 11.759 & 630 \\
\hline $\mathrm{P} 210^{6} \quad(\mathrm{~kg})$ & 17.068 & $186,72(28,48)$ & 490 & 9.699 & 534 \\
\hline P3657 (kg) & 13.973 & $250,39(39,84)$ & 473 & 8.121 & 556 \\
\hline $\mathrm{P} 450^{8} \quad(\mathrm{~kg})$ & 11.957 & $298,47 \quad(46,63)$ & 451 & 6.896 & 443 \\
\hline $\mathrm{P} 550^{9} \quad(\mathrm{~kg})$ & 4.254 & $347,14(63,55)$ & 249 & 3.096 & 201 \\
\hline
\end{tabular}

1 Circunferência escrotal padronizada aos 365 dias de idade (Standardized scrotal circumference at 365 days of age).

2 Circunferência escrotal padronizada aos 450 dias de idade (Standardized scrotal circumference at 450 days of age).

Circunferência escrotal padronizada aos 550 dias de idade (Standardized scrotal circumference at 550 days of age).

Altura do posterior ao sobreano (Hip height at 18 month of age).

Peso padronizado aos 120 dias de idade (Standardized weight at 120 days of age).

Peso padronizado aos 210 dias de idade (Standardized weight at 210 days of age).

Peso padronizado aos 365 dias de idade (Standardized weight at 365 days of age).

Peso padronizado aos 450 dias de idade (Standardized weight at 450 days of age).

9 Peso padronizado aos 550 dias de idade (Standardized weight at 550 days of age).

10 Grupo de contemporâneos (Contemporary group). 
Foram assumidas as seguintes pressuposições: $\mathrm{E}(y)=$ $X \beta, \mathrm{E}(a)=0, \mathrm{E}(m)=0, \mathrm{E}(e)=0$, e:

$\operatorname{Var} \quad\left(\begin{array}{c}a \\ m \\ c \\ e\end{array}\right)=\left(\begin{array}{cccc}A \sigma^{2} & 0 & 0 & 0 \\ 0 & A \sigma^{2} \mathrm{~m} & 0 & 0 \\ 0 & 0 & I \sigma_{\mathrm{c}}^{2} & 0 \\ 0 & 0 & 0 & I \sigma_{\mathrm{e}}\end{array}\right)$

em que $A$ é a matriz do numerador dos coeficientes de parentesco entre os animais; $I$, uma matriz identidade; $\sigma^{2}{ }_{\mathrm{a}}$, $\sigma_{\mathrm{m}}^{2}, \sigma_{\mathrm{C}}^{2}$ e $\sigma_{\mathrm{e}}^{2}$, as variâncias dos efeitos aleatórios para $a, m, c$ e $e$, respectivamente.

O modelo para as características P120, P210 e P365 incluiu o efeito aleatório genético direto, materno e de ambiente permanente materno e o efeito fixo do GC e da classe da idade da vaca ao parto (CIVP). A idade da vaca ao parto foi dividida em seis classes constituídas por fêmeas que pariram até os 35 meses, de 36 a 47, 48 a 59, 60 a 71, 72 a 119 meses e acima de 120 meses de idade. A covariância genética entre os efeitos aditivo direto e materno foi fixada em zero, como sugerido por Meyer (1997), Benyshek et al. (1998) e Albuquerque \& Meyer (2001). As características P450 e P550 foram analisadas por modelos semelhantes ao modelo [1], desconsiderando-se, em ambos os casos, o efeito aleatório de ambiente permanente materno. Adicionalmente, para P550, foram excluídos do modelo o efeito aleatório genético materno e o efeito fixo da CIVP. A característica ALT foi analisada por um modelo que incluiu o efeito aleatório genético direto; o GC, como fixo; e o efeito linear da idade do animal na mensuração (IDA, variando de 450 a 599 dias) e os efeitos linear e quadrático da idade da vaca ao parto (IVP, variando de 23 a 283 meses), como covariáveis.

As circunferências escrotais padronizadas foram analisadas por dois modelos, o primeiro incluía o efeito aleatório genético direto, o efeito fixo do GC e o peso padronizado nas respectivas idades (efeitos linear e quadrático), como covariável, enquanto o segundo excluía a covariável peso padronizado. Após, calculou-se a correlação de Spearman, nas respectivas idades, entre os valores genéticos preditos para circunferência escrotal, obtidos em ambos os modelos (utilizando ou não o peso como covariável), considerando dois conjuntos dados, um com todos os animais e outro com apenas touros pais.

\section{Resultados e Discussão}

As estimativas de herdabilidade apresentaram errospadrão que variaram de 0,02 a 0,09. Essas herdabilidades são de amplitudes moderadas a altas, indicando que essas características devem responder rapidamente à seleção massal (Tabela 2).
Tabela 2 - Estimativas de herdabilidades do efeito direto (diagonal), correlações genéticas (acima da diagonal) e correlações de ambiente (abaixo da diagonal) para características de crescimento em bovinos da raça Nelore

Table 2 - Direct effect heritability (diagonal), genetic correlation (above the diagonal) and environmental correlation (below the diagonal) estimates for growth traits in Nelore cattle

\begin{tabular}{llllll}
\hline $\begin{array}{l}\text { Característica } \\
\text { Trait }\end{array}$ & ALT & P210 & P365 & P450 & P550 \\
\hline $\mathrm{ALT}^{1}(\mathrm{~cm})$ & 0,63 & 0,64 & 0,53 & 0,58 & 0,59 \\
$\mathrm{P}^{2} 10^{2}(\mathrm{~kg})$ & 0,35 & 0,34 & 0,94 & 0,95 & 0,90 \\
$\mathrm{P}^{3} 5^{3}(\mathrm{~kg})$ & 0,49 & 0,73 & 0,45 & 0,99 & 0,98 \\
$\mathrm{P}^{4} 0^{4}(\mathrm{~kg})$ & 0,49 & 0,61 & 0,85 & 0,48 & 1,00 \\
$\mathrm{P}_{50}(\mathrm{~kg})$ & 0,43 & 0,68 & 0,73 & 0,84 & 0,49 \\
\hline
\end{tabular}

${ }^{1}$ Altura do posterior ao sobreano (Hip height at 18 moth of age).

2 Peso padronizado aos 210 dias de idade (Standardized weight at 210 days of age).

3 Peso padronizado aos 365 dias de idade (Standardized weight at 365 days of age)

4 Peso padronizado aos 450 dias de idade (Standardized weight at 450 days of age)

5 Peso padronizado aos 550 dias de idade (Standardized weight at 550 days of age).

Para os pesos padronizados aos 120, 210, 365 e 450 dias de idade, as estimativas da herdabilidade do efeito materno e seus respectivos erros-padrão foram de $0,11(0,02), 0,10$ $(0,02), 0,09(0,03)$ e $0,08(0,03)$, respectivamente. As proporções entre as variâncias de ambiente permanente materno e fenotípicas e seus respectivos erros-padrão para o P120, P210 e P365 foram de 0,15 (0,02), 0,17 (0,03) e 0,04 (0,02), respectivamente. Resultados semelhantes às estimativas de herdabilidade dos efeito diretos e materno obtidos neste trabalho foram descritos por diversos autores para a raça Nelore (Reyes et al., 1997; Siqueira et al., 2003; Costa et al., 2004). Koots et al. (1994), Mercadante et al. (1995) e Lobo et al. (2000), em trabalhos de revisão bibliográfica, relataram resultados semelhantes de estimativas de herdabilidade dos efeitos direto e materno, para peso em diversas idades, variando de 0,08 a 0,44 .

Altas estimativas de herdabilidade para altura do posterior, como as encontrada neste trabalho, têm sido descritas na literatura (Vargas et al., 1998, 2000), em animais zebuínos com idades próximas ao sobreano. Poucos trabalhos foram encontrados para animais da raça Nelore, entretanto, Cyrillo et al. (2001), Silva et al. (2003) e Pereira et al. (2004) estimaram herdabilidades de amplitude moderada à alta, variando de 0,27 a 0,58, em idades próximas ao ano, sobreano e desmame, respectivamente.

As estimativas de herdabilidade para as circunferências escrotais em diferentes idades foram de magnitudes altas (Tabelas 2, 3 e 4), sendo que a inclusão do peso como covariável no modelo de análise não afetou as estimativas aos 365 e 450 dias de idade. Contudo, aos 550 dias, a estimativa passou de 0,49 para 0,42 ; provavelmente, esta redução ocorreu devido ao fato de os pesos às diferentes idades serem geneticamente correlacionados à CE, como 
Tabela 3 - Estimativas de herdabilidade do efeito direto (diagonal), correlações genéticas (acima da diagonal) e correlações de ambiente (abaixo da diagonal) entre características reprodutivas e de crescimento, em análises trivariadas com o P120 e ajustando as circunferências escrotais para peso, em bovinos da raça Nelore

Table 3 - Direct effect heritability (diagonal), genetic correlation (above the diagonal) and environmental correlation (below the diagonal) estimates between reproductive and growth traits using three trait models including $W 120$ and scrotal circumference adjusted for weight, in Nelore cattle

\begin{tabular}{lrrrrr}
\hline $\begin{array}{l}\text { Característica } \\
\text { Trait }\end{array}$ & ALT & P120 & CE365 & CE450 & CE550 \\
\hline ALT $^{1}(\mathrm{~cm})$ & 0,63 & 0,68 & $-0,19$ & $-0,24$ & 0,00 \\
P120 $^{2}(\mathrm{~kg})$ & 0,36 & 0,25 & $-0,08$ & $-0,03$ & 0,13 \\
CE365 $^{3}(\mathrm{~mm})$ & $-0,04$ & 0,09 & 0,48 & 0,96 & 0,76 \\
CE450 $^{4}(\mathrm{~mm})$ & 0,18 & 0,11 & 0,72 & 0,53 & 0,91 \\
CE550 $^{5}(\mathrm{~mm})$ & 0,13 & 0,17 & 0,46 & 0,71 & 0,42 \\
\hline
\end{tabular}

1 Altura do posterior ao sobreano (Hip height at 18 month of age).

2 Peso padronizado aos 120 dias de idade (Standardized weight at 120 days of age).

${ }^{3}$ Circunferência escrotal padronizada aos 365 dias de idade (Standardized scrotal circumference at 365 days of age).

4 Circunferência escrotal padronizada aos 450 dias de idade (Standardized scrotal circumference at 450 days of age).

${ }^{5}$ Circunferência escrotal padronizada aos 550 dias de idade (Standardized scrotal circumference at 550 days of age).

observado na Tabela 4 e em outros trabalhos (Alencar et al., 1993; Bergmann et al., 1996; Cyrillo et al., 2001). Assim, incluindo-se o peso no modelo, perdeu-se parte da variabilidade genética da característica CE. Semelhantemente ao observado para CE550, Quirino \& Bergman (1997), estudando animais da raça Nelore, verificaram que as variâncias genéticas e ambientais e as herdabilidades estimadas para circunferência escrotal aos 9, 12, 18 e 24 meses de idade diminuíram quando o peso do animal foi incluído no modelo de análise. Por outro lado, Dias et al. (2003), também na raça Nelore, relataram estimativas de herdabilidade superiores para esta característica, quando peso e idade foram considerados no modelo. Neste caso, os autores observaram que a inclusão do peso no modelo diminuiu a variância residual e não a genética. Estimativas de herdabilidade para CE em animais Nelore, nessas respectivas idades, com magnitudes próximas às encontradas neste estudo, foram descritas por Cyrillo et al. (2001), Costa et al. (2004), Albuquerque et al. (2005) e Sezana (2005).

As herdabilidades estimadas para a CE em diferentes idades (365, 450 e 550 dias) sugerem que o uso da CE450 como critério de seleção poderá proporcionar maior resposta à seleção. Provavelmente, aos 365 dias de idade, poucos animais já entraram na puberdade, ao passo que, aos 550 dias de idade, a maior parte desses animais já deve ter atingido este estádio de desenvolvimento, o que, de alguma forma, pode diminuir as diferenças genéticas entre os animais. Contudo, para definição da idade mais adequada para mensuração da CE, além de se considerar a variabilidade genética da característica, é necessário estimar as correlações desta com características de precocidade sexual e reprodução das fêmeas. Estimativas de herdabilidade das CE em diferentes idades, semelhantes às encontradas neste trabalho, para animais da raça Nelore, foram descritas por Albuquerque et al. (2005), que estimaram maiores herdabilidades para a circunferência escrotal entre 400 e 480 dias de idade, por meio da regressão aleatória. Entretanto, Sezana (2005) não observou grandes diferenças nas estimativas de herdabilidade para CE aos 12, 16, 18 e 21 meses de idade, que foram de 0,43 a 0,45 , porém, aos 9 meses de idade, notou-se considerável diminuição da variabilidade genética, sendo a estimativa de herdabilidade de 0,22. Diferenças nas estimativas de herdabilidade descritas na literatura podem ocorrer não só pelas diferenças nas estruturas dos dados, mas também pelos modelos de análise utilizados, entre outros fatores.

Entre a ALT e os pesos padronizados em diferentes idades, as correlações genéticas (Tabelas 2 e 3) foram positivas e de magnitudes moderadas $(0,53$ a 0,64). Em animais da raça Brahman, Vargas et al. (2000) estimaram correlação genética entre ALT e peso ao desmame um pouco superior ao valor encontrado neste trabalho $(0,75)$. Associação genética entre peso e altura aos 378 dias de idade, próxima à obtida nesta pesquisa, também na raça Nelore, de 0,61, foi estimada por Cyrillo et al. (2001). Assim, a seleção para peso, pode, em médio ou longo prazo, conduzir a aumento na estrutura dos animais. Entretanto, é possível a obtenção de animais com maior peso e altura moderada, uma vez que a correlação genética entre essas características não é próxima à unidade. Contudo, para que isso ocorra, é preciso que estas características sejam consideradas simultaneamente em programas de melhoramento genético animal.

As correlações genéticas obtidas entre os pesos em diferentes idades foram positivas e acima de 0,90 (Tabela 2), indicando que, provavelmente, grande parte dos mesmos conjuntos de genes de ação aditiva atua na expressão destas características. Progressos genéticos para pesos em diferentes idades podem ser alcançados pelas suas respostas correlacionadas. Assim, o produtor poderia utilizar, como critério de seleção, um peso em uma idade que permita avaliação da habilidade maternal (P120 ou P210) e outro peso na idade mais próxima ao período de comercialização, seja na desmama, ao ano ou sobreano, dependendo do seu objetivo. Robertson (1959) sugeriu que, quando a correlação genética entre duas características estiver abaixo de 0,80, ambas devem ser consideradas nos programas de seleção. 
Tabela 4 - Estimativas de herdabilidade do efeito direto e correlações genéticas $\left(\hat{r}_{g}\right)$ e ambientais $\left(\hat{r}_{e}\right)$, em análises trivariada utilizando o P120, entre as características reprodutivas e de crescimento, sendo as circunferências escrotais em diferentes idades sem ajustar para os pesos, em bovinos da raça Nelore

Table 4 - Direct effect heritability, genetic correlation $\left(\hat{r}_{g}\right)$ and environmental correlation ( $\hat{r}_{e}$ ) estimates between reproductive and growth trait using three trait models including W120 and scrotal circumference not adjusted for weight, in Nelore cattle

\begin{tabular}{|c|c|c|c|c|c|c|}
\hline $\begin{array}{l}\text { Característica } \\
\text { Trait }\end{array}$ & \multicolumn{2}{|c|}{$\mathrm{CE} 365^{3}(\mathrm{~mm})$} & \multicolumn{2}{|c|}{$\mathrm{CE} 450^{4}(\mathrm{~mm})$} & \multicolumn{2}{|c|}{$\mathrm{CE} 550^{5}(\mathrm{~mm})$} \\
\hline Correlação & $\hat{r}_{g}$ & $\hat{r}_{e}$ & $\hat{r}_{g}$ & $\hat{r}_{e}$ & $\hat{r}_{g}$ & $\hat{r}_{e}$ \\
\hline \multicolumn{7}{|l|}{ Correlation } \\
\hline $\mathrm{ALT}^{1}(\mathrm{~cm})$ & 0,21 & 0,13 & 0,12 & 0,33 & 0,39 & 0,20 \\
\hline $\mathrm{P} 120^{2}(\mathrm{~kg})$ & 0,57 & 0,35 & 0,51 & 0,33 & 0,53 & 0,34 \\
\hline $\begin{array}{l}\text { Herdabilidade } \\
\text { Heritability }\end{array}$ & \multicolumn{2}{|c|}{0,48} & \multicolumn{2}{|c|}{0,54} & \multicolumn{2}{|c|}{0,49} \\
\hline \multicolumn{7}{|c|}{$\begin{array}{l}1 \text { Altura do posterior ao sobreano (Hip height at } 18 \text { month of age). } \\
2 \text { Peso padronizado aos } 120 \text { dias de idade (Standardized weight at } 120 \text { days of age). } \\
{ }^{3} \text { Circunferência escrotal padronizada aos } 365 \text { dias de idade (Standardized } \\
\text { scrotal circumference at } 365 \text { days of age). } \\
4 \text { Circunferência escrotal padronizada aos } 450 \text { dias de idade (Standardized } \\
\text { scrotal circumference at } 450 \text { days of age). } \\
5 \text { Circunferência escrotal padronizada aos } 550 \text { dias de idade (Standardized } \\
\text { scrotal circumference at } 550 \text { days of age). }\end{array}$} \\
\hline
\end{tabular}

A correlação genética entre CE e peso foi positiva e moderada (Tabela 4), uma vez que a CE foi ajustada somente para a idade, resultados semelhante aos obtidos na raça Nelore (Bergmann et al., 1996; Cyrillo et al., 2001; Costa et al., 2004). Como esperado, quando as circunferências escrotais foram corrigidas para peso, essa correlação foi próxima de zero (Tabela 3). Essas correlações genéticas mostram que a seleção para o aumento da CE deve resultar, por resposta correlacionada, em aumento do peso, caso a CE não seja ajustada para o peso corporal.

As associações genéticas entre a ALT e as circunferências escrotais ajustadas para peso nas três diferentes idades foram negativas e próximas de zero (Tabela 3). Entretanto, quando as circunferências escrotais não foram ajustadas para o peso, essas estimativas foram positivas e de magnitudes baixas a moderadas (Tabela 4). Vargas et al. (1998), estudando animais da raça Brahman, próximos aos 550 dias de idade, relataram estimativa de correlação genética entre essas características positiva e próxima de zero $(0,19)$. Estas correlações (ALT e CE e Peso) sugerem que seleção para CE não ajustada para o peso deve acarretar aumento do peso e da altura do posterior.

As estimativas de correlações genéticas entre as CE ajustadas (Tabela 3) ou não para o peso, nas três diferentes idades, foram positivas e altas. As maiores correlações foram em idades mais próximas, sendo um pouco mais elevadas, de 0,96; 0,81 e 0,92, quando não se ajustaram as circunferências escrotais para o peso, respectivamente, entre CE365 x CE450, CE365 x CE550 e CE450 x CE550. Na literatura, constam poucos trabalhos sobre correlações genéticas entre CE em diferentes idades, em animais da raça Nelore. Bergmann et al. (1996) estimaram correlação genética inferior à deste trabalho $(0,76)$ entre a CE365 e CE550. Correlações genéticas positivas e altas entre as CE nas diferentes idades, decrescendo com o aumento da distância em idade entre as medidas, também foram obtidas por Albuquerque et al. (2005), utilizando regressão aleatória. Da mesma forma, Sezana (2005) descreveu estimativas de correlações genéticas entre as circunferências escrotais nas idades de 9, 12, 15, 18 e 21 meses variando de 0,76 a 0,97. Em animais da raça Canchim, Alencar et al. (1993) relataram estimativas de correlações genéticas entre as circunferências escrotais, medidas próximas aos 365, 550 e 730 dias de idade, positivas e acima de 0,68 , com correlações mais altas quando as medidas foram feitas em idades mais próximas, conforme encontrado nesta pesquisa.

As correlações de Spearman entre os valores genéticos da circunferência escrotal em uma mesma idade, obtidos por ambos os modelos, de todos os animais com dados, foram altas, sendo de 0,81 para CE365 e 0,98 para CE450 e CE550. Quando se consideraram apenas os valores genéticos dos touros, estas correlações foram de 0,75 (CE365), 0,97 (CE450) e 0,98 (CE550). Estes resultados indicam que, para CE aos 450 e 550 dias de idade, a inclusão do peso no modelo de análise, praticamente, não deve mudar a classificação dos animais ou dos touros. Entretanto, aos 365 dias de idade, as mudanças de classificação podem ser importantes e a definição de quais covariáveis incluir no modelo deve ser baseada no objetivo de seleção.

\section{Conclusões}

Os parâmetros genéticos estimados neste trabalho indicam que as características de crescimento e circunferência escrotal apresentam variabilidade genética na raça Nelore, podendo ser incluídas em programas de melhoramento genético e devendo responder bem à seleção individual. É possível selecionar animais com maiores circunferências escrotais nas diferentes idades utilizando apenas a circunferência escrotal mensurada próxima aos 450 dias de idade. A seleção para circunferência escrotal não ajustada para peso deve levar à resposta correlacionada no mesmo sentido, para as características de crescimento. Seleção para peso nas diferentes idades deve resultar em aumento da altura do posterior por resposta correlacionada. Na busca por animais pesados, com estatura moderada, os 
resultados sugerem que é necessário compor um índice econômico aliando estas características (peso e altura).

\section{Literatura Citada}

ALBUQUERQUE, L.G.; MEYER, K. Estimates of direct and maternal genetic effects for weights from birth to 600 days of age in Nelore cattle. Journal of Animal Breeding and Genetic, v.118, p.83-92, 2001.

ALBUQUERQUE, L.G.; SILVA, J.A.V.; SESANA, R.C. et al. Estimativas de parâmetros genéticos para perímetro escrotal na raça Nelore, usando regressão aleatória. In: REUNIÓN DE LA ASOCIACIÓN LATINOAMERICANA DE PRODUCCIÓN ANIMAL, 19., 2005, Tampico. Anais... Tampico: Asociación Latinoamericana de Producción Animal, 2005. (CD-ROM).

ALENCAR, M.M.; BARBOSA, P.F.; BARBOSA, R.T. et al. Parâmetros genéticos para peso e circunferência escrotal em touros da raça Canchim. Revista da Sociedade Brasileira Zootecnia, v.22, n.4, p.572-583, 1993.

BAKER, J.F.; STEWART, T.S.; LONG, C.R. et al. Multiple regression and principal components analysis of puberty and growth in cattle. Journal of Animal Science, v.66, p.2147-2158, 1998.

BEEF IMPROVEMENT FEDERATION - BIF. Guidelines for uniform beef improvement programs. 7.ed. Manhattan: Kansas State University, 1996. p.17-20.

BENYSHEK, L.L.; JOHNSON, M.H.; LITTLE, D.E. et al. Application of an animal model in the United States beef cattle industry. Journal of Dairy Science, v.71, p.35-53, 1988 (supl. 2).

BERGMANN, J.A.G.; ZAMBORLINI, L.C.; PROCÓPIO, C.S.O. et al. Estimativas de parâmetros genéticos do perímetro escrotal e do peso corporal em animais da raça Nelore. Arquivo Brasileiro de Medicina Veterinária e Zootecnia, v.48, p.69-78, 1996.

BOLDMAN, K.G.; KRIESE, L.A.; Van VLECK, L.D. et al. A manual for use of MTDFREML. A set of programs to obtain estimates of variances and covariances [DRAFT]. Lincoln: U. S. Department of Agriculture, Agricultural Research Service, 1995. 120p.

COSTA, B.R.; LAUREANO, M.M.M.; FORNI, S. et al. Estimativas de parâmetros genéticos para as características perímetro escrotal, peso ao sobreano e idade ao primeiro parto em um rebanho da raça Nelore. In: SIMPÓSIO DA SOCIEDADE BRASILEIRA DE MELHORAMENTO ANIMAL, 5., 2004, Pirassununga. Anais... Pirassununga: Sociedade Brasileira de Melhoramento Animal, 2004. (CD-ROM).

CYRILLO, J.N.S.G.; RAZOOK A.G.; FIGUEIREDO, L.A. et al. Estimativas de tendências e parâmetros genéticos do peso padronizado aos 378 dias de idade, medidas corporais e perímetro escrotal de machos nelore de Sertãozinho, SP. Revista Brasileira de Zootecnia, v.30, n.1, p.56-65, 2001.

DIAS L.T.; EL FARO L.; ALBUQUERQUE L.G. Estimativas de herdabilidade para perímetro escrotal de animais da raça Nelore. Revista Brasileira Zootecnia, v.32, n.6, p.1878-1882, 2003. (supl. 2).

DIAS, L.T.; FARO, L.E.; FRIES, L.A. et al. Estimativas de parâmetros genéticos para perímetro escrotal e idade ao primeiro parto em animais da raça Nelore. In: REUNIÃO ANUAL DA SOCIEDADE BRASILEIRA DE ZOOTECNIA, 37., 2000, Viçosa, MG. Anais... Viçosa, MG: Sociedade Brasileira de Zootecnia, 2000. (CD-ROM).

KNIGHTS, S.A.; BAKER, R.L.; GIANOLA, D. et al. Estimates of heritabilities and of genetic and phenotypic correlations among growth and reproductive traits in yearling Angus bulls. Journal of Animal Science, v.58, n.4, p.887-893, 1984.

KOOTS, K.R.; GIBSON, J.P.; SMITH, C. et al. Analyses of published genetic parameters estimates for beef production traits. 1. Heritability. Animal Breeding Abstracts, v.62, p.309-338, 1994.
LOBO, R.N.B.; MADALENA, F.E.; VIEIRA, A.R. Average estimates of genetic parameters for beef and dairy cattle in tropical regions. Animal Breeding Abstracts, v.68, n.6, p.433-462, 2000.

MATTOS, S.; ROSA, A.N. Desempenho reprodutivo de fêmeas das raças zebuínas. Informativo Agropecuário, v.10, n.112, p.2933, 1984.

MERCADANTE, M.E.Z.; LÔBO, R.B.; BORJAS, A.R. Parámetros genéticos para características de crecimiento en cebuínos de carne. Archivos Latinoamericanos de Produccion Animal, v.3, p.45-89, 1995.

MEYER, K. Estimates of genetic parameters for weaning weight of beef cattle accounting for direct-maternal environmental covariances. Livestock Production Science, v.52, p.187-199, 1997.

PATTERSON, H.D.; THOMPSON, R. Recovery of inter-block information when block size are unequal. Biometrics, v.58, p.545-554, 1971.

PEREIRA, E.; ELER, J.P.; FERRAZ, J.B.S. Correlação genética entre perímetro escrotal e algumas características reprodutivas na raça Nelore. Revista Brasileira de Zootecnia, v.29, n.6, p.1676-1683, 2000.

PEREIRA, E.; ELER, J.P.; FERRAZ, J.B.S. Análise genética de características reprodutivas na raça Nelore. Pesquisa Agropecuária Brasileira, v.37, n.5, p.703-708, 2002.

PEREIRA, M.C.; YOKOO, M.J.; BIGNARDI A.B. et al. Estimativas de parâmetros genéticos e de ambiente para a altura à desmama em bovinos da raça Nelore. In: SIMPÓSIO DA SOCIEDADE BRASILEIRA DE MELHORAMENTO ANIMAL, 5., 2004, Pirassununga. Anais... Pirassununga: Sociedade Brasileira de Melhoramento Animal, 2004. (CD-ROM).

QUIRINO, C.R.; BERGMANN, J.A. Herdabilidade do perímetro escrotal padronizado e não padronizado para peso corporal usando modelo animal uni e bivariado. In: REUNIÃO ANUAL DA SOCIEDADE BRASILEIRA DE ZOOTECNIA, 34., 1997, Juiz de Fora. Anais... Juiz de Fora: Sociedade Brasileira de Zootecnia, 1997. p.127-129.

REYES, A. de LOS; LÔBO, R.B.; BEZERRA, L.A.F. et al. Variabilidade genética de características de crescimento alternativas para a seleção em gado de corte. In: REUNIÃO ANUAL DA SOCIEDADE BRASILEIRA DE ZOOTECNIA, 34. 1997, Juiz de Fora. Anais... Juiz de Fora: Sociedade Brasileira de Zootecnia, 1997. p.245-247.

RILEY, D.G.; CHASE JR, C.C.; HAMMOND, A.C. et al. Estimated genetic parameters for carcass traits of Brahman cattle. Journal of Animal Science, v.80, p.955-962, 2002.

ROBERTSON, A. The sampling variance of the genetic correlation coefficient. Biometrics, v.15, p.469-485, 1959.

STATISTICAL ANALYSIS SYSTEM - SAS. SAS/STAT Software: changes and enhancement through release 8.2. Cary: 2000. (CD-ROM).

SESANA, R.C. Estimativas de parâmetros genéticos para perímetro e volume escrotal, medidos em diferentes idades em animais da raça Nelore. Jaboticabal: Universidade Estadual Paulista, 2005. 40p. Trabalho de Iniciação Científica (Graduação em Zootecnia) - Universidade Estadual Paulista, 2005.

SILVA, A.M.; ALENCAR, M.M.; FREITAS, A.R. et al. Herdabilidades e correlações genéticas para peso e perímetro escrotal de machos e características reprodutivas e de crescimento de fêmeas, na raça Canchim. Revista Brasileira de Zootecnia, v.29, n.6, p.2223-2230, 2000 (supl. 2).

SILVA, J.A. II V.; Van MELIS, M.H.; ELER, J.P. et al. Estimação de parâmetros genéticos para probabilidade de prenhez aos 14 meses e altura na garupa em bovinos da raça Nelore. Revista Brasileira de Zootecnia, v.32, n.5, p.1141-1146, 2003.

SILVA, J.A. II V.; ALBUQUERQUE, L.G.; SESANA, R.C. et al. Correlação genética entre prenhez de novilhas e perímetro escrotal em animais Nelore utilizando inferência Bayesiana In: REUNIÓN DE LA ASOCIACIÓN LATINOAMERICANA DE PRODUCCIÓN ANIMAL, 19., 2005, Tampico. Anais... 
Tampico: Asociación Latinoamericana de Producción Animal, 2005. (CD-ROM).

SIQUEIRA, R.L.P.G.; OLIVEIRA J.A.; LÔBO R.B. et al. Análise da variabilidade genética aditiva de características de crescimento na raça Nelore. Revista Brasileira Zootecnia, v.32, n.1, p.99105, 2003.

VARGAS, C.A.; ELZO M.A.; CHASE, JR., C.C. et al. Estimation of parameters for scrotal circumference, age at puberty in heifers, and hip height in Brahman cattle. Journal of Animal Science, v.76, p.2536-2541, 1998.
VARGAS, C.A.; ELZO M.A.; CHASE, JR., C.C. et al. Genetic parameters and relationships between hip height and weight in Brahman cattle. Journal of Animal Science, v.78, p.30453052, 2000.

Recebido: 10/4/2006 Aprovado: 31/5/2007 